\title{
On the number of distributive lattices
}

\author{
Marcel Erné, Jobst Heitzig, and Jürgen Reinhold \\ Institut für Mathematik, Universität Hannover, \\ Welfengarten 1, D-30167 Hannover, Germany \\ \{erne,heitzig,reinhold\}@math.uni-hannover.de
}

Submitted: March 2, 2001; Accepted: April 1, 2002.

MR Subject Classifications: 05A15, 05A16, 06A07, $06 \mathrm{D} 05$.

Key words: canonical poset, distributive lattice, ordinal (vertical) decomposition.

\begin{abstract}
We investigate the numbers $d_{k}$ of all (isomorphism classes of) distributive lattices with $k$ elements, or, equivalently, of (unlabeled) posets with $k$ antichains. Closely related and useful for combinatorial identities and inequalities are the numbers $v_{k}$ of vertically indecomposable distributive lattices of size $k$. We present the explicit values of the numbers $d_{k}$ and $v_{k}$ for $k<50$ and prove the following exponential bounds:

$$
1.67^{k}<v_{k}<2.33^{k} \text { and } 1.84^{k}<d_{k}<2.39^{k} \quad\left(k \geqslant k_{0}\right) .
$$

Important tools are (i) an algorithm coding all unlabeled distributive lattices of height $n$ and size $k$ by certain integer sequences $0=z_{1} \leqslant \cdots \leqslant z_{n} \leqslant k-2$, and (ii) a "canonical 2-decomposition" of ordinally indecomposable posets into "2indecomposable" canonical summands.
\end{abstract}

\section{Vertical decompositions and additive functions}

For the enumeration of classes of finite posets or lattices, so-called ordinal resp. vertical decompositions are of particular use (see, for example, $[6,7]$ ). Roughly speaking, ordinal and vertical summation consists of placing the posets "above" each other, perhaps identifying extremal elements. As we are mainly interested in unlabeled (i.e. isomorphism classes of) posets and lattices, it suffices here to give the formal definitions only for sufficiently disjoint ground sets: The ordinal sum of two posets $P_{1}=\left(X_{1}, \sqsubseteq_{1}\right)$ and $P_{2}=\left(X_{2}, \sqsubseteq_{2}\right)$ with (o) $X_{1} \cap X_{2}=\emptyset$ can be defined as $P_{1} \oplus P_{2}=\left(X_{1} \cup X_{2}\right.$, $)$, where

$$
x \sqsubseteq y \Longleftrightarrow x \sqsubseteq_{1} y \text { or } x \sqsubseteq_{2} y \text { or }(x, y) \in X_{1} \times X_{2} .
$$

Although this is also defined for lattices, one rather considers the vertical sum in that case, where the only difference to the former is that now the top element $T_{1}$ of the lower summand and the bottom element $\perp_{2}$ of the upper summand are identified instead of 
becoming neighbours: If $L_{1}=\left(X_{1}, \sqsubseteq_{1}\right)$ and $L_{2}=\left(X_{2}\right.$, 2$)$ are lattices with (v) $X_{1} \cap X_{2}=$ $\left\{\top_{1}\right\}=\left\{\perp_{2}\right\}$, their vertical sum can be formally defined as the lattice $L=\left(X_{1} \cup X_{2}\right.$, $)$ with $\sqsubseteq$ as above. The ordinal [vertical] sum of two isomorphism classes is of course the isomorphism class of the sum of two representatives that fulfill (o) [(v)].

Now, a poset [lattice] is ordinally [vertically] decomposable if it is either empty [a singleton] or the ordinal [vertical] sum of two nonempty posets [non-singleton lattices], otherwise it is ordinally [vertically] indecomposable. The following facts are well known and easily verified.

Lemma 1 Ordinal and vertical summation are associative (but clearly not commutative). Every finite poset [lattice] has a unique ordinal [vertical] decomposition into ordinally [vertically] indecomposable posets [lattices]. Vertical components of a lattice are intervals of that lattice.

For graph theorists it may be of interest that the ordinal decomposition of a poset into indecomposable summands corresponds to the partition of the incomparability graph into connected components.

By Birkhoff's Theorem [3], the unlabeled finite posets are in one-to-one correspondence with the homeomorphism classes of finite $T_{0}$ spaces [1] and also with the unlabeled finite distributive lattices, by assigning to each poset $P$ its topology (hence distributive lattice) $\mathcal{A}(P)$ of all lower sets (also known as downsets, decreasing sets, lower segments, order ideals). On the other hand, the latter are just the complements of upper sets (also known as upsets, increasing sets, upper segments, order filters), and each upper, resp. lower set is generated by a unique antichain (in the finite case). Therefore, the cardinalities of the following entities are counted by the same number $d_{k}$ :

- unlabeled distributive lattices with $k$ elements,

- non-homeomorphic $T_{0}$ spaces with $k$ open (closed) sets,

- unlabeled posets with $k$ antichains (upper sets, lower sets).

The above one-to-one correspondence does not preserve ordinal sums, but instead sends the ordinal sum of $P$ and $Q$ to the vertical sum of $\mathcal{A}(P)$ and $\mathcal{A}(Q)$. Therefore, the same symbol $v_{k}$ may denote the number of all

- vertically indecomposable unlabeled distributive lattices with $k$ elements,

- non-homeomorphic $T_{0}$ spaces having no nonempty proper open subset comparable to all other open sets,

- ordinally indecomposable unlabeled posets with $k$ antichains, upper sets, or lower sets, respectively.

From Lemma 1, we infer immediately (cf. [6, 7]):

Corollary 2 The numbers $v_{k}$ are related to the numbers $d_{k}$ by

$$
d_{1}=1, v_{1}=0, \text { and } d_{k}=\sum_{j=1}^{k-1} v_{k-j+1} d_{j} \text { for } k \geqslant 2 \text {. }
$$




\section{A useful representation of finite distributive lattices}

We shall use a special case of A. Day's "doubling construction" [4], generating larger lattices from given ones. Let $D=(k, \sqsubseteq)$, be a distributive lattice of height $n$, where we adopt the usual set-theoretic definition of natural numbers $k=\{0,1, \ldots, k-1\}$. Consider an element $z \in D$ and the principal filter $I=\uparrow z:=\{d \in D: z \sqsubseteq d\}$. Let $\psi: I^{\uparrow} \rightarrow I$ be the unique isomorphism from the distributive lattice $I^{\uparrow}$ with underlying set $\{k, \ldots, k+|I|-1\}$ onto $I$ such that $\psi$ is strictly increasing with respect to the usual order $\leqslant$ on the natural numbers. Define the order relation $\sqsubseteq^{\uparrow}$ on $k+|I|$ by

$$
\begin{aligned}
& x \sqsubseteq \uparrow y \\
& x, y<k \text { and } x \sqsubseteq y \\
& \text { or } \quad x, y \geqslant k \text { and } \psi(x) \sqsubseteq \psi(y) \\
& \text { or } \quad x<k \leqslant y \text { and } x \sqsubseteq \psi(y) \text {. }
\end{aligned}
$$

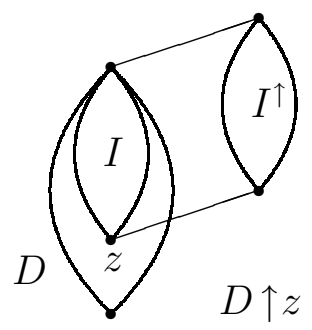

Then $D \uparrow z:=\left(k+|I|, \sqsubseteq^{\uparrow}\right)$ is again a distributive lattice, and $D$ is a retract of $D \uparrow z$ with retraction $y \mapsto y \wedge \bigvee D\left(=\psi(y)\right.$ for $\left.y \in I^{\uparrow}\right)$. This construction reflects the extensions of the corresponding poset $P$ of $\vee$-irreducible (equivalently: $\vee$-prime) elements by one new maximal point $n$ (see [5]): the join map from $\mathcal{A}(P)$ to $D$ is an isomorphism, and for any $Z \in \mathcal{A}(P)$, there is a unique poset $P \cup\{n\}$ containing $P$ as a subposet such that $n$ becomes a maximal element generating the principal ideal $Z \cup\{n\}$. Now, the above isomorphism extends to one between $\mathcal{A}(P \cup\{n\})$ and $D \uparrow z$ where $z=\bigvee Z$. Any isomorphism $\varphi: D \rightarrow D^{\prime}=\left(k, \sqsubseteq^{\prime}\right)$ extends uniquely to an isomorphism $\varphi^{\uparrow}$ between $D \uparrow z$ and $D^{\prime} \uparrow \varphi(z)$ (mapping $y \in \uparrow k$ to $\varphi^{\uparrow}(y)=\psi^{\prime-1} \circ \varphi \circ \psi(y)$ ).

Since every poset of size $n+1$ arises from one of size $n$ by the one-point extension process described above, every finite distributive lattice with more than one element is isomorphic to one of the form $D \uparrow z$. Directly, this can also be seen as follows. Any $\wedge$-prime element $x$ in a finite distributive lattice $E$ has a unique cover $u$, and there is a least element $y$ not dominated by $x$. This $y$, henceforth denoted by $u \backslash x$, in turn is $\vee$-prime and covers a unique element $z$. The intervals $[z, x]$ and $[y, u]$ of $E$ are isomorphic via transposition: $z=x \wedge y, u=x \vee y$. Moreover, $E$ is the disjoint union of $\downarrow x=\{e \in E: e \sqsubseteq x\}$ and $\uparrow y=\{e \in E: y \sqsubseteq e\}$. Now, it is easy to verify that if $x$ is a coatom in $E$ and $D$ is the principal ideal $\downarrow x$ then the whole lattice $E$ is isomorphic to $D \uparrow z$.

This observation makes it possible to generate any finite distributive lattice up to isomorphism by a finite number of "doublings" of principal filters.

Theorem 3 Every distributive lattice $(D, \sqsubseteq)$ of finite cardinality $k>1$ and height $n$ is isomorphic to a lattice of the form $D_{0} \uparrow z_{1} \uparrow \ldots \uparrow z_{n}$ with $\left|D_{0}\right|=1$ and a sequence $\left(z_{1}, \ldots, z_{n}\right) \in k^{n}$ with $0=z_{1} \leqslant z_{2} \leqslant \cdots \leqslant z_{n}$. 
Figure 1: A handy network of distributive lattices of size $\leqslant 8$ or height $\leqslant 4$

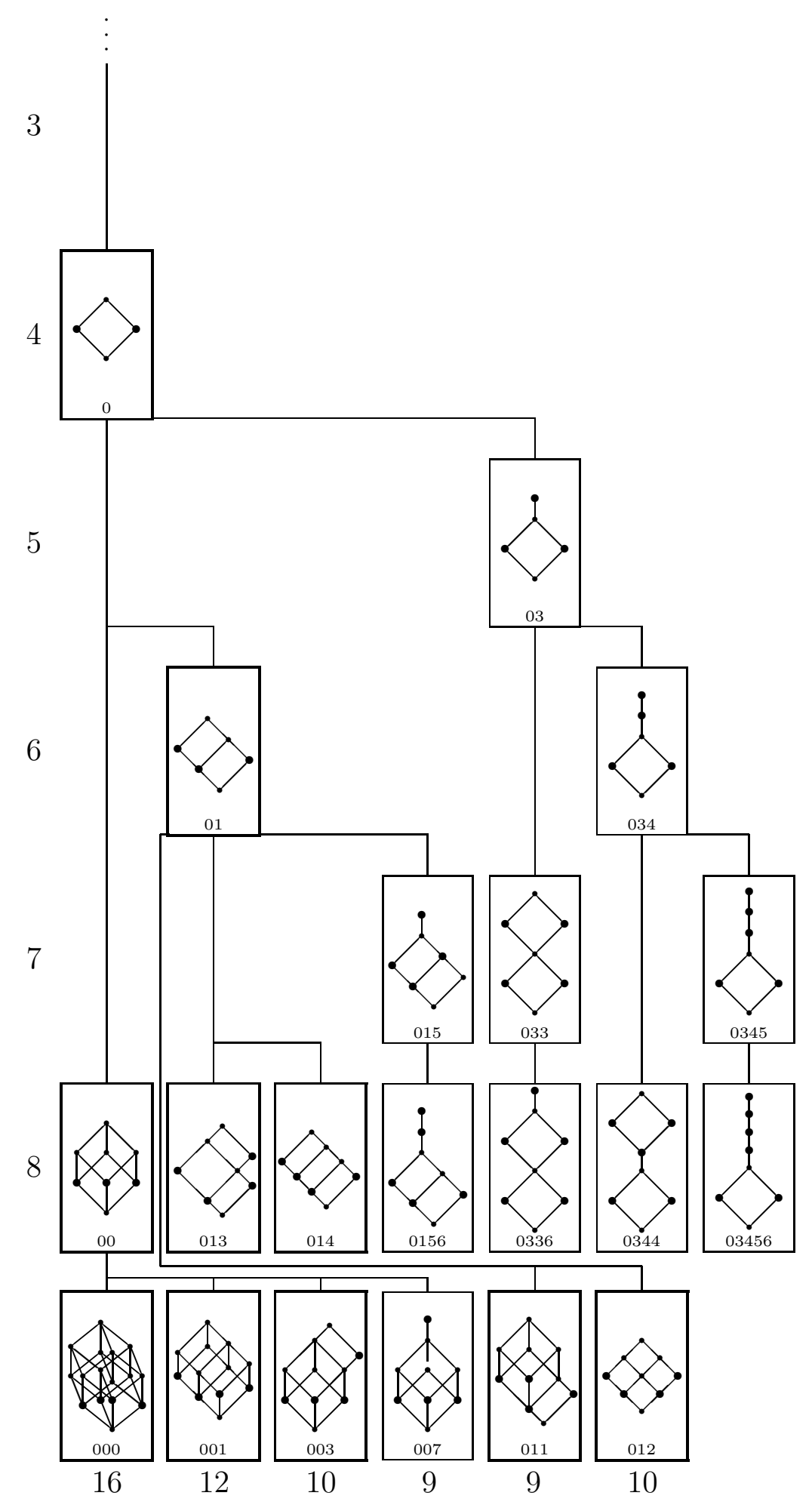


Figure 2: A handy network of distributive lattices (continued)

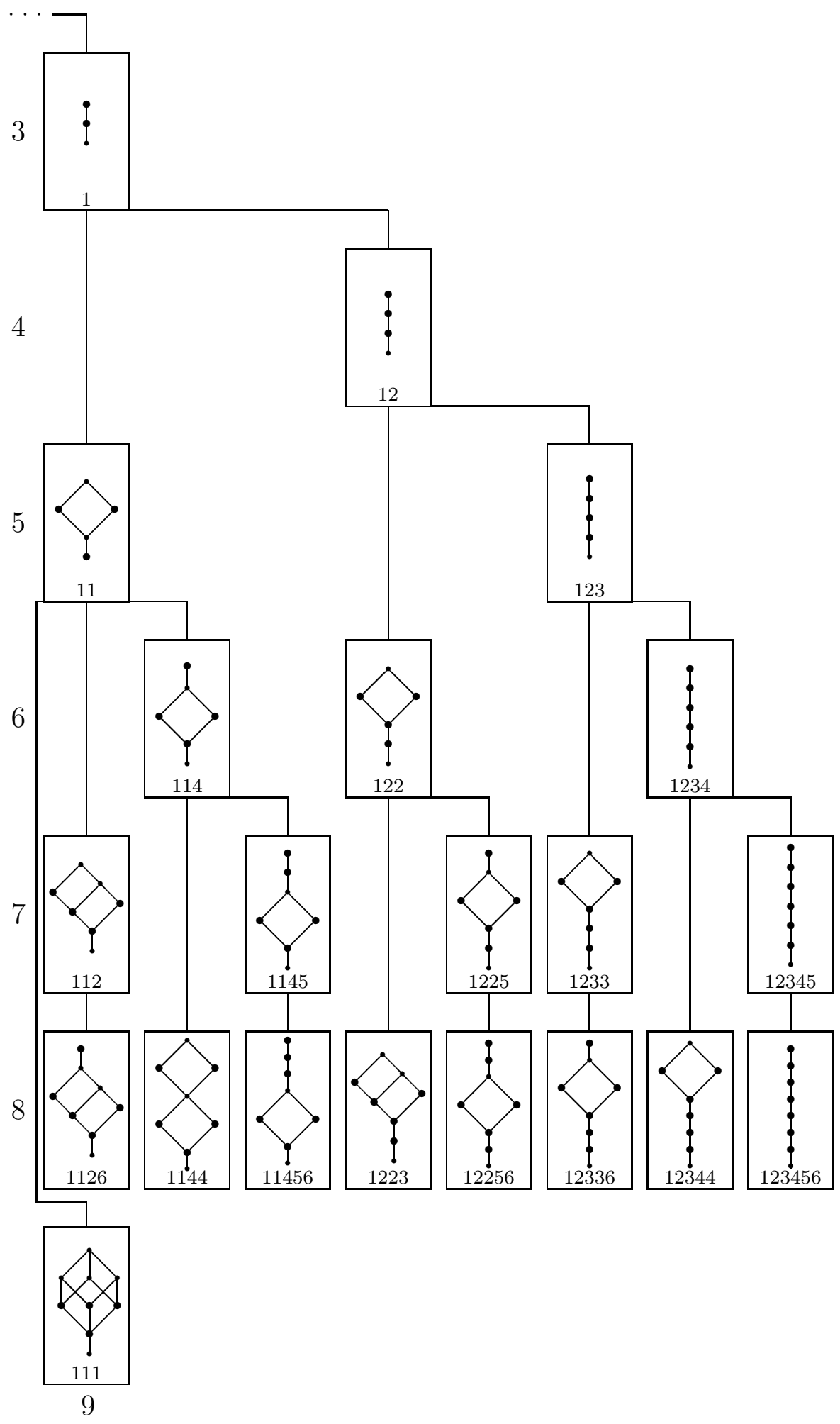


Proof. We recursively determine elements $x_{i}, y_{i}, z_{i} \in D$, distributive lattices $D_{i}=\left(k_{i}, \sqsubseteq_{i}\right)$ and isomorphisms $\varphi_{i}: \downarrow x_{i} \rightarrow D_{i}$, so that $x_{0} \sqsubset x_{1} \sqsubset \cdots \sqsubset x_{n}$ is a maximal chain in $D$, $y_{1}, \ldots, y_{n}$ are the $\vee$-irreducible elements of $D, z_{1}, \ldots, z_{n}$ are their unique lower covers, $u \sqsubseteq v$ implies $\varphi_{i}(u) \leqslant \varphi_{i}(v)$ (in the natural order), $\varphi_{i}$ extends $\varphi_{i-1}$, and $D_{i}=D_{i-1} \uparrow$ $\varphi_{i-1}\left(z_{i}\right)(i>0)$.

Let $x_{0}=y_{0}=z_{0}$ be the bottom element and $D_{0}$ the distributive lattice with underlying set $1=\{0\}$. Then $\varphi_{0}: \downarrow x_{0} \rightarrow D_{0}$ is uniquely determined. If $x_{i-1}, y_{i-1}, z_{i-1}$ and $\varphi_{i-1}$ have been defined and $x_{i-1}$ is not the top of $D$, take for $x_{i}$ one element among those covers $u$ of $x_{i-1}$ for which $\varphi_{i-1}\left(x_{i-1} \wedge\left(u \backslash x_{i-1}\right)\right)$ is minimal in the natural order $\leqslant$ on $D_{i-1}$, and put $y_{i}=x_{i} \backslash x_{i-1}, z_{i}=x_{i-1} \wedge y_{i}$. Then $y_{i}$ is $\vee$-irreducible and $z_{i}$ is its unique lower cover. Moreover, the intervals $\left[z_{i}, x_{i-1}\right]$ and $\left[y_{i}, x_{i}\right]$ are isomorphic via transposition, and $\downarrow x_{i}=\downarrow x_{i-1} \cup\left[y_{i}, x_{i}\right]$. Hence, there exists an isomorphism $\varphi_{i}: \downarrow x_{i} \rightarrow D_{i}=D_{i-1} \uparrow \varphi_{i-1}\left(z_{i}\right)$ satisfying $u \sqsubseteq v \Rightarrow \varphi_{i}(u) \leqslant \varphi_{i}(v)$ and extending $\varphi_{i-1}$. Continuing the construction, we get an isomorphism $\varphi=\varphi_{n}$ between $D$ and $D_{n}=D_{0} \uparrow \varphi\left(z_{1}\right) \uparrow \ldots \uparrow \varphi\left(z_{n}\right)$.

Thus, we see that $D$ is uniquely determined, up to isomorphism, by the sequence $\varphi\left(z_{1}\right), \ldots, \varphi\left(z_{n}\right)$. Without loss of generality, let $\varphi$ be the identity map. Finally, we show that the sequence $0=z_{1}, \ldots, z_{n}$ is increasing. Assume $i<j$ but $z_{j}<z_{i}$. Since $z_{j}$ is covered by $y_{j}$ and $y_{j} \nsubseteq x_{i-1} \sqsubset x_{j-1}$, it follows that $x_{i-1}=z_{j} \vee x_{i-1}$ is covered by $x_{i}{ }^{\prime}:=y_{j} \vee x_{i-1}$. Moreover, in the interval $\downarrow x_{i}$,

$$
y_{i}^{\prime}:=x_{i}{ }^{\prime} \backslash x_{i-1}=\min \left\{d \in \downarrow x_{i}{ }^{\prime}: d \nsubseteq x_{i-1}\right\} \sqsubseteq y_{j}
$$

and $z_{i}{ }^{\prime}:=x_{i-1} \wedge y_{i}{ }^{\prime} \sqsubseteq x_{j-1} \wedge y_{j}=z_{j}$, whence $z_{i}{ }^{\prime} \leqslant z_{j}<z_{i}$, contradicting the choice of $x_{i}$ (making $z_{i}$ minimal).

Notice that in the above theorem several different sequences (e.g. $(0,0,1)$ and $(0,0,2))$ may describe the same isomorphism type, and that not every increasing sequence $\left(z_{1}, \ldots, z_{n}\right) \in k^{n}$ corresponds to a distributive lattice. For example, it is not difficult to see that the construction yields the following inequality:

Corollary 4 If an integer sequence $z_{1} \leqslant \cdots \leqslant z_{n}$ represents a distributive lattice $D_{0} \uparrow$ $z_{1} \uparrow \ldots \uparrow z_{n}$ then

$$
\sum_{i=1}^{j} z_{i}<2^{j-1} \text { for } 1 \leqslant j \leqslant n \text {, in particular } z_{1}=0 .
$$

Proof. The lattices $D_{i}=\downarrow x_{i}=D_{0} \uparrow z_{1} \uparrow \ldots \uparrow z_{i}$ have height $i$ and, therefore, size $k_{i} \leqslant 2^{i}$. Furthermore, $k_{0}=1$ and $k_{i}=\left|\downarrow x_{i-1}\right|+\left|\left[z_{i}, x_{i-1}\right]\right| \leqslant 2 k_{i-1}-z_{i}$ for $i>0$. Hence, $z_{i} \leqslant 2 k_{i-1}-k_{i}$ and

$$
\sum_{i=1}^{j} z_{i} \leqslant 2 \sum_{i=1}^{j} k_{i-1}-\sum_{i=1}^{j} k_{i}=2+\sum_{i=1}^{j-1} k_{i}-k_{j} \leqslant 1+\sum_{i=1}^{j-2} k_{i}<2^{j-1} .
$$

Another inequality immediately results from doubling one- or two-element intervals only: 
Corollary 5 The number $d_{k}$ of distributive lattices with $k$ elements is greater than or equal to the $k$-th Fibonacci number $F_{k}$ (with $F_{1}=0$ and $F_{2}=1$ ).

The previous construction may be used to generate a set of representatives (coded by finite sequences of natural numbers) for the isomorphism classes of finite distributive lattices with at least two elements. Define recursively such representative $d$-sequences as follows. The empty sequence is a representative d-sequence (for the 2-element chain). Assume $\left(z_{2}, \ldots, z_{n-1}\right)$ is a representative $\mathrm{d}$-sequence, representing a distributive lattice $D=D_{0} \uparrow z_{1} \uparrow \ldots \uparrow z_{n-1}$. If $k$ is the size of $D$ then for each integer $z$ with $z_{n-1} \leqslant$ $z \leqslant k-1$, the sequence $\left(z_{2}, \ldots, z_{n-1}, z\right)$ codes the distributive lattice $D \uparrow z$. Now, call $\left(z_{2}, \ldots, z_{n-1}, z_{n}\right)$ a representative d-sequence if $z_{n}$ is minimal among all $z$ for which $D \uparrow z$ is isomorphic to $D \uparrow z_{n}$. By our earlier remarks on the doubling construction, this selects from each isomorphism class of finite distributive lattices one representative which is coded by the (increasing) sequence $\left(z_{2}, \ldots, z_{n}\right)$. Indeed, if $D$ is any distributive lattice of height $n$ and size $k$ then $D$ is isomorphic to $D_{0} \uparrow z_{1} \uparrow \ldots \uparrow z_{n}$ for some sequence(s) of natural numbers $z_{1}=0, z_{2}, \ldots, z_{n}$. Taking the lexicographically smallest among these sequences, one obtains a representative d-sequence (proof by induction, using the unique extensions of isomorphisms from $D_{i-1}$ to $D_{i}=D_{i-1} \uparrow z_{i}$ ). Similarly, one checks that different representative d-sequences represent non-isomorphic lattices. Figures 1 and 2 show how all distributive lattices with $\leqslant 8$ elements or height $\leqslant 4$ arise in this way, the vertically indecomposable ones being framed by bold lines.

\section{A second ordinal decomposition of a poset}

In this section we need a notion of canonicity adopted from $[8,9]$ which is useful for various kinds of ordered structures. For the sake of consistency with the forerunners, we prefer here a downward numbering of elements. Of course, an upward numbering would work as well.

Here, an $n$-poset is a poset $P$ with underlying set $n=\{0, \ldots, n-1\}$. We write $i \prec j$ if $j$ is a cover of $i$ in $P$ and define the weight

$$
w_{P}=\left(w_{P}(0), \ldots, w_{P}(n-1)\right)
$$

of an $n$-poset $P$ by setting

$$
w_{P}(i)=\sum_{i \prec j} 2^{j} .
$$

Since a finite poset is uniquely determined by its covering relation, the map $P \mapsto w_{P}$ is injective. Let $P, Q$ be $n$-posets. Then we say that $w_{P}$ is (lexicographically) smaller than $w_{Q}$ if there is an $i \leqslant n-1$ such that $w_{P}(i)<w_{Q}(i)$ and $w_{P}(k)=w_{Q}(k)$ for all $k=0, \ldots, i-1$. We call an $n$-poset $C$ a canonical poset if there is no $n$-poset isomorphic to $C$ that has a smaller weight. It was shown in $[8,9]$ that for every canonical $n$-poset $C$ the sequence $w_{C}$ is increasing, i.e. $w_{C}(0) \leqslant \cdots \leqslant w_{C}(n-1)$.

The set $P_{1}$ of all maximal elements in a finite poset $P$ is called the first level of $P$. One recursively defines the $i$-th level $P_{i}$ of $P$ to be the first level of the subposet $P \backslash \bigcup_{j=1}^{i-1} P_{j}$. 
It is well known and easy to see that an element $x \in P$ is contained in $P_{i}$ iff $i$ is the maximal cardinality of a chain in $P$ with least element $x$, denoted by $d_{P}(x)$ (the depth of $x)$. Notice that $x \sqsubset y$ implies $d_{P}(x)>d_{P}(y)$. The height of the poset $P$ will be denoted by $h(P)$. The last nonempty level $\left\{x \in P: d_{P}(x)=h(P)+1\right\}$ consists of minimal elements only, but there may also be minimal elements of $P$ in higher levels. It was proven in $[8,9]$ that every canonical poset $P$ is level-monotone (= "levelized" in the cited papers), i.e. $d_{P}(x) \leqslant d_{P}(y)$ for all $x, y \in P$ with $x \leqslant y$.

Let $p, q$ be natural numbers and let $P=\left(p, \sqsubseteq_{P}\right), Q=\left(q, \sqsubseteq_{Q}\right)$ be canonical posets. Set

$$
\begin{aligned}
& p^{+q}=(p+q) \backslash q=\{q, q+1, \ldots, q+p-1\}, \\
& \sqsubseteq_{P}^{+q}=\left\{(x+q, y+q): x \sqsubseteq_{P} y\right\}, \\
& P^{+q}=\left(p^{+q}, \sqsubseteq^{+q}\right), \\
& \sqsubseteq=\sqsubseteq_{Q} \cup \sqsubseteq_{P}^{+q} \cup\left(p^{+q} \times q\right), \\
& \sqsubseteq_{2}=\sqsubseteq \backslash\{(q, q-1)\} .
\end{aligned}
$$

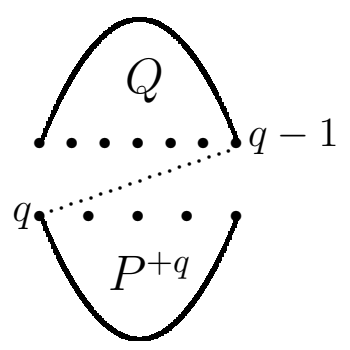

Since $P$ and $Q$ are level-monotone, the element $q-1$ is minimal in $Q$ and $q$ is maximal in $P^{+q}$. Now, it is easy to verify that $\sqsubseteq$ and $\sqsubseteq_{2}$ are order relations on $p+q$. Also, it is not hard to see that the "canonical sum" $(p+q, \sqsubseteq)$ is the canonical representative for the ordinal sum $P \oplus Q$. More involved is the proof of the following property of the "canonical 2-sum" $P+{ }_{2} Q:=\left(p+q, \sqsubseteq_{2}\right)$.

Theorem 6 If $P=\left(p, \sqsubseteq_{P}\right)$ and $Q=\left(q, \sqsubseteq_{Q}\right)$ are ordinally indecomposable canonical posets then $\mathrm{R}=\mathrm{P}+{ }_{2} Q$ is also an ordinally indecomposable canonical poset.

Proof. Let $\varphi$ be a permutation of $p+q$ such that the poset $R^{\prime}=\left(p+q,\left\{(x, y): \varphi(x) \sqsubseteq_{2}\right.\right.$ $\varphi(y)\})$ is canonical. In order to prove that $R$ is canonical, we have to verify that the vector $w_{R^{\prime}}=\left(w_{R^{\prime}}(0), \ldots, w_{R^{\prime}}(p+q-1)\right)$ coincides with $w_{R}=\left(w_{R}(0), \ldots, w_{R}(p+q-1)\right)$, i.e., that $\varphi$ is an automorphism of $R$.

Let $t, \ldots, q-1$ be the minimal elements in $Q$ and let $q, \ldots, q+s$ be the maximal elements in $P^{+q}$. We shall only consider the case $t<q-1$, i.e. that $Q$ has at least two minimal elements. Otherwise, it would follow from the ordinal indecomposability of $Q$ that it has only one element. In that case some of the weights below have to be computed in a different way but the reader may easily check that all arguments stay correct. Since $P$ and $Q$ are canonical, they are level-monotone. Then $R$ is also levelmonotone since $d_{R}(x)=d_{Q}(x)$ for $x \in q$ and $d_{R}(y)=d_{P}(y)+h(Q)+1$ for $y \in p^{+q} \backslash\{q\}$, while $d_{R}(q) \in\{h(Q)+1, h(Q)+2\}$. 
If $d_{R}(q)=h(Q)+2$ then the fact that the canonical poset $R^{\prime}$ is also level-monotone implies that $\varphi[q]=q$ and, since $Q$ is canonical, that $\left.\varphi\right|_{q}$ is an automorphism of $Q$, i.e. $w_{R^{\prime}}(x)=w_{R}(x)$ for $x \in q$. Then

$$
w_{R^{\prime}}\left(\varphi^{-1}(q)\right)=\sum_{i=t}^{q-1} 2^{i}-2^{\varphi^{-1}(q-1)}<\sum_{i=t}^{q-1} 2^{i} \leqslant w_{R^{\prime}}(y)
$$

for every element $y \in p^{+q} \backslash\left\{\varphi^{-1}(q)\right\}$. Since $R^{\prime}$ is canonical, $w_{R^{\prime}}$ is increasing and, therefore, $\varphi(q)=q$. Now,

$$
w_{R^{\prime}}(q)=\sum_{i=t}^{q-1} 2^{i}-2^{\varphi^{-1}(q-1)} \geqslant \sum_{i=t}^{q-2} 2^{i}=w_{R}(q)
$$

implies $\varphi(q-1)=q-1$ and, therefore, $w_{R^{\prime}}(q)=w_{R}(q)$.

If $d_{R}(q)=h(Q)+1$ then $\varphi[q+1]=q+1$. In this case, $\{q-1\}$ is the last level of $Q$ and $\{q-1, q\}$ constitutes a whole level in $R$ and in $R^{\prime}$. Since all covers of $q-1$ dominate $q$ in $R$, it follows from the minimality of $w_{R^{\prime}}$ that $\varphi(q-1)=q-1$ and $\varphi(q)=q$. Again, we see that $\left.\varphi\right|_{q}$ is an automorphism of $Q$ and $w_{R^{\prime}}(q)=\sum_{i=t}^{q-2} 2^{i}=w_{R}(q)$.

Since either $\{q, \ldots, q+s\}$ or $X:=\{q+1, \ldots, q+s\}$ is one level of $R$ and of $R^{\prime}$ (or empty) and since $\varphi(q)=q$, we have $\varphi[X]=X$. All elements $x \in X$ have the same covers in $R$ and $R^{\prime}$, namely $t, \ldots, q-1$, i.e. $w_{R^{\prime}}(x)=\sum_{i=t}^{q-1} 2^{i}=w_{R}(x)$ for $x \in X$.

Let $s+1, \ldots, s+u$ be those elements in $P$ which are covered by 0 only. Then $w_{R}(y)=2^{q-1}+2^{q}$ for $y \in Y:=\{q+s+1, \ldots, q+s+u\}$ and $w_{R}(z) \geqslant 2^{q+1}$ for $z \in Z:=\{q+s+u+1, \ldots, q+p-1\}$. Notice that for $z \in Z$, every cover of $z$ in $R$ or $R^{\prime}$ is contained in $p^{+q}$. From the lexicographic minimality of $w_{R^{\prime}}$ it follows that $\varphi[Y]=Y$ and that $w_{R^{\prime}}(y)=w_{R}(y)$ for $y \in Y$.

Consider the poset $\tilde{P}=\left(p,\left\{(x, y): \varphi(x+q) \sqsubseteq_{2} \varphi(y+q)\right\}\right)$. If $w_{R^{\prime}}$ were lexicographically smaller than $w_{R}$ then the vector

$$
\begin{aligned}
w_{\tilde{P}} & =\left(w_{\tilde{P}}(0), \ldots, w_{\tilde{P}}(s), w_{\tilde{P}}(s+1), \ldots, w_{\tilde{P}}(s+u), w_{\tilde{P}}(s+u+1), \ldots, w_{\tilde{P}}(p-1)\right) \\
& =\left(0, \ldots, 0,1, \ldots, 1,2^{-q} w_{R^{\prime}}(q+s+u+1), \ldots, 2^{-q} w_{R^{\prime}}(q+p-1)\right)
\end{aligned}
$$

would be lexicographically smaller than

$$
w_{P}=\left(0, \ldots, 0,1, \ldots, 1,2^{-q} w_{R}(q+s+u+1), \ldots, w_{R}(q+p-1)\right),
$$

contradicting the canonicity of $P$.

Now, in order to prove that $R$ is ordinally indecomposable, let us assume the contrary. Then there is a nonempty proper upper set $S$ of $R$ such that the relation $((p+q) \backslash S) \times S$ is contained in $\Xi_{2}$. Since $q \rrbracket_{2} q-1$, we have $S \neq q$, whence $S \nsubseteq q$ or $q \nsubseteq S$. In the first case, $S \cap p^{+q}$ is a nonempty proper upper subset in $P^{+q}$ with $\left(p^{+q} \backslash S\right) \times\left(S \cap p^{+q}\right) \subseteq \sqsubseteq_{P}{ }^{+q}$, i.e., $P^{+q}$ and $P$ are ordinally decomposable. In the second case, $S \cap q$ is a nonempty proper upper set of $Q$ and $(q \backslash S) \times(S \cap q) \subseteq \sqsubseteq_{Q}$, i.e. $Q$ is ordinally decomposable, a contradiction. 
The above theorem says that $+_{2}$ is an operation on the set of ordinally indecomposable canonical posets. It is not difficult to check from the definition that this operation is associative. If the canonical posets $P=\left(p, \sqsubseteq_{P}\right), Q=\left(q, \sqsubseteq_{Q}\right)$ have $i$ and $j$ antichains, respectively, then $P+{ }_{2} Q$ has $i+j$ antichains because every nonempty antichain of $P+{ }_{2} Q$ different from $\{q-1, q\}$ is either contained in $Q$ or in $P^{+q}$, while the empty antichain is contained in both.

An ordinally indecomposable canonical poset $R$ will be called canonically 2decomposable if there are ordinally indecomposable canonical posets $P, Q$ with $R=P+{ }_{2} Q$. We denote by $w_{k}$ the number of canonically 2 -indecomposable posets with $k$ antichains.

If $R=\left(r, \sqsubseteq_{R}\right)$ is an ordinally indecomposable but canonically 2-decomposable poset then there is a smallest $p<r$ such that there are ordinally indecomposable posets $P=\left(p, \sqsubseteq_{P}\right), Q=\left(q, \sqsubseteq_{Q}\right)$ with $R=P+_{2} Q$. Then, clearly, $P$ and $Q$ are unique, and associativity of $+_{2}$ assures that $P$ is canonically 2 -indecomposable. Hence the number of those posets which are ordinally indecomposable but canonically 2-decomposable, have $k$ antichains, and whose first canonically 2-indecomposable summand has exactly $i$ antichains, is $w_{i} \cdot v_{k-i}$. Since a nonempty poset has at least 2 antichains, it follows that

$$
v_{k}=w_{k}+\sum_{i=2}^{k-2} w_{i} \cdot v_{k-i}
$$

Corollary 7 The numbers $w_{k}$ of canonically 2-indecomposable posets with $k$ antichains are related to the numbers $v_{k}$ of ordinally indecomposable posets with $k$ antichains by the identities

$$
v_{0}=1, \quad w_{1}=v_{1}=0, \quad \text { and } \quad v_{k}=\sum_{j=0}^{k-1} w_{k-j} \cdot v_{j} \quad(k>1) .
$$

It would be reasonable to call a poset (ordinally) 2-indecomposable if it is indecomposable and augmenting the order relation by one arbitrary pair never produces a decomposable poset. The number of such posets with $k$ antichains is, of course, at most $w_{k}$. But, unfortunately, not every 2-decomposable poset is canonically 2-decomposable (consider the disjoint union of a singleton and a 3-chain) and, what is more important, there is no formula like that in the previous corollary for 2 -indecomposable posets. A poset is 2-indecomposable if its incomparability graph is 2-edge-connected.

\section{Exponential estimates for summatorial sequences}

This section contains the necessary theoretical background for the intended (partly asymptotical) estimates of the numbers $d_{k}$ and $v_{k}$. In what follows, $\left(a_{k}: k \geqslant 1\right)$ always designates a sequence of nonnegative real numbers, and

$$
a(x)=\sum_{k=1}^{\infty} a_{k} x^{k} \quad \text { and } \quad a_{<m}(x)=\sum_{k=1}^{m-1} a_{k} x^{k}
$$


the corresponding (formal) power series and its partial sums, regarded as polynomials. The "summatorial" sequence $\left(s_{k}\right)$ and its partial sums are given by

$$
s(x)=\sum_{k=0}^{\infty} s_{k} x^{k}=(1-a(x))^{-1}=\sum_{k=0}^{\infty} a(x)^{k}, \quad s_{<m}(x)=\sum_{k=0}^{m-1} s_{k} x^{k}
$$

and their coefficients are determined recursively by

$$
s_{0}=1, \quad s_{k}=\sum_{j=0}^{k-1} a_{k-j} s_{j}=\sum_{j=1}^{k} a_{j} s_{k-j} \quad \text { for } k \geqslant 1 .
$$

We say that a proposition holds "eventually" when it holds for all $k$ larger than some $k_{0}$.

Lemma 8 The following statements are equivalent:

(1) $s_{k}>0$ eventually.

(2) There is no integer $m>1$ with $a_{k}>0 \Longrightarrow m \mid k$.

(3) $\operatorname{gcd}\left(m: a_{m}>0\right)=1$.

Proof. (1) $\Longrightarrow(2)$ : If $m \mid k$ for all $k$ with $a_{k}>0$ then the recursion for $s_{k}$ yields $s_{k}=0$ whenever $m \nmid k$.

$(2) \Longrightarrow(3)$ : Clear.

$(3) \Longrightarrow(1)$ : There exist indices $k_{1}, \ldots, k_{u}$ with $\operatorname{gcd}\left(k_{1}, \ldots, k_{u}\right)=1$ and $a_{k_{i}}>0$ for $i=1, \ldots, u$. Hence, for each natural number $k$, there are integers $l_{1}, \ldots, l_{u}$ with $k_{1} l_{1}+$ $\cdots+k_{u} l_{u}=k$, and if $k$ is sufficiently large, then the $l_{i}$ can be chosen nonnegative, whence

$$
s_{k} \geqslant a_{k_{1}} s_{k-k_{1}} \geqslant a_{k_{1}}^{2} s_{k-2 k_{1}} \geqslant \cdots \geqslant \prod_{i=1}^{u} a_{k_{i}}^{l_{i}}>0
$$

where we used the recursion formula $l_{1}+\cdots+l_{u}$ times.

In the subsequent lemmas, we always assume that (1)-(3) are fulfilled. Lower exponential bounds for $s_{k}$ are provided by

Lemma 9 Suppose $m \in \mathbb{N}$ and $\sigma>0$ are constants with $a_{<m}\left(\frac{1}{\sigma}\right)>1$. Then there is a $\tau>\sigma$ and an $n$ with $m \leqslant n<2 m$ and $s_{k} \tau^{-k} \geqslant s_{n} \tau^{-n}$ for all $k \geqslant m$. Hence, if $s_{k}>0$ for $k \geqslant m$,

$$
\tau^{k}=O\left(s_{k}\right) \quad \text { and } \quad \sigma^{k}=o\left(s_{k}\right) .
$$

Proof. By continuity, there is a $\tau>\sigma$ with $a_{<m}\left(\frac{1}{\tau}\right)>1$. Put $\delta:=\min \left\{s_{j} \tau^{-j}: m \leqslant j<\right.$ $2 m\}$, say $\delta=s_{n} \tau^{-n}$. Then $s_{j} \tau^{-j} \geqslant \delta$ for all $j$ with $m \leqslant j<2 m$. Let $k \geqslant 2 m$ and assume that $s_{j} \tau^{-j} \geqslant \delta$ has also been established for all $j$ with $m \leqslant j<k$. Then

$$
s_{k} \geqslant \sum_{j=1}^{m-1} a_{j} s_{k-j} \geqslant \sum_{j=1}^{m-1} a_{j} \delta \tau^{k-j}=\delta \tau^{k} a_{<m}\left(\frac{1}{\tau}\right)>\delta \tau^{k} .
$$


Hence, by induction, $s_{k} \tau^{-k} \geqslant \delta=s_{n} \tau^{-n}$ for all $k \geqslant m$.

Let $\varrho_{s}$ denote the radius of convergence for $s(x)$. If the series $1-a(x)$ has a smallest positive root $\varrho$, then $\varrho=\varrho_{s}$, since by nonnegativity of the $a_{k}$ and monotonicity of $a(x)$, the series $s(x)$ surely converges for $0 \leqslant x<\varrho$ and diverges for $x>\varrho$.

The criterion in Lemma 9 is not only sufficient but also necessary for the estimate $\sigma^{k}<\tau^{k}=O\left(s_{k}\right)$. More precisely:

Corollary 10 For $\sigma>0$, the following statements are equivalent:

(a) $a\left(\frac{1}{\sigma}\right)>1$ (not excluding $\left.a\left(\frac{1}{\sigma}\right)=\infty\right)$.

(b) $a_{<m}\left(\frac{1}{\sigma}\right)>1$ for some $m$.

(c) $\tau^{k}=O\left(s_{k}\right)$ for some $\tau>\sigma$.

(d) For some $\tau^{\prime}>\sigma$, eventually $\sqrt[k]{s_{k}}>\tau^{\prime}$.

(e) $\limsup \sqrt[k]{s_{k}}>\sigma$.

Proof. $(\mathrm{a}) \Longleftrightarrow$ (b) is clear since $\sup _{m} a_{<m}\left(\frac{1}{\sigma}\right)=a\left(\frac{1}{\sigma}\right)$ (at least improperly).

(b) $\Longrightarrow$ (c) follows from Lemma 9 .

For $(\mathrm{c}) \Longrightarrow(\mathrm{d})$, choose $\varepsilon>0$ with $\tau^{k} \leqslant \varepsilon s_{k}$ for all $k$, a $\tau^{\prime}$ with $\sigma<\tau^{\prime}<\tau$, and finally an $n$ with $\varepsilon<\left(\frac{\tau}{\tau^{\prime}}\right)^{n}$; then each $k \geqslant n$ satisfies $\tau \leqslant \sqrt[k]{\varepsilon s_{k}}<\frac{\tau}{\tau^{\prime}} \sqrt[k]{s_{k}}$, hence $\tau^{\prime}<\sqrt[k]{s_{k}}$.

$(\mathrm{d}) \Longrightarrow(\mathrm{e}): \lim \sup \sqrt[k]{s_{k}} \geqslant \tau^{\prime}>\sigma$.

$(\mathrm{e}) \Longrightarrow(\mathrm{a}):$ Since $\varrho_{s}=\left(\limsup \sqrt[k]{s_{k}}\right)^{-1}<\sigma^{-1}$, there is an $x<\sigma^{-1}$ for which $s(x)$ diverges. Thus, it cannot happen that $a(x)$ converges to a value $<1$, because otherwise $s(x)=(1-a(x))^{-1}$ were convergent. It follows that $a\left(\frac{1}{\sigma}\right)>a(x) \geqslant 1$.

Interestingly, the implication $(\mathrm{e}) \Longrightarrow(\mathrm{d})$ shows that the limes superior of the values $\sqrt[k]{s_{k}}$ is in fact a proper limit:

Corollary $11 \sqrt[k]{s_{k}}$ converges to $\frac{1}{\varrho_{s}}$.

As another consequence of Corollary 10, we get

Corollary 12 If $a\left(\frac{1}{\sigma}\right)>1$ for some $\sigma>0$ then $\sigma^{k}=o\left(s_{k}\right)$.

Now we derive upper exponential bounds for $s_{k}$ from those for $a_{k}$.

Lemma 13 Suppose there are constants $m \in \mathbb{N}, \gamma>0$, and $\sigma>\alpha>0$ such that

(1) $a_{k} \leqslant \gamma \alpha^{k}$ for $k \geqslant m$,

(2) $a_{<m}\left(\frac{1}{\sigma}\right)+\gamma\left(\frac{\alpha}{\sigma}\right)^{m} \frac{\sigma}{\sigma-\alpha}<1$.

Then there is a $\tau$ with $\alpha<\tau<\sigma$ and

The ELECTRONiC JOURNAL OF COMBINATORICS 9 (2002), \#R24 
(3) $s_{k}=O\left(\tau^{k}\right)$, a fortiori $s_{k}=o\left(\sigma^{k}\right)$.

If, in addition,

(4) $\alpha^{m-1}(\sigma-\alpha) s_{<m}\left(\frac{1}{\alpha}\right) \leqslant s_{m}$

then there exists an integer $n$ with $m \leqslant n<2 m$ and

(5) $s_{k} \tau^{-k} \leqslant s_{n} \tau^{-n}$ for all $k \geqslant m$.

Proof. By continuity, there is a $\tau$ with $\alpha<\tau<\sigma$ such that (2) holds for $\tau$ instead of $\sigma$. Put

$$
\delta:=\max \left\{\frac{\tau-\alpha}{\alpha}\left(\frac{\alpha}{\tau}\right)^{m} s_{<m}\left(\frac{1}{\alpha}\right), s_{j} \tau^{-j}: m \leqslant j<2 m\right\} .
$$

Then $s_{j} \leqslant \delta \tau^{j}$ for $m \leqslant j<2 m$. Consider a $k \geqslant 2 m$ such that $s_{j} \leqslant \delta \tau^{j}$ for all $j$ with $m \leqslant j<k$. Then, by $(1)$,

$$
\begin{aligned}
s_{k} & =\sum_{j=0}^{k-1} a_{k-j} s_{j} \leqslant \gamma \sum_{j=0}^{m-1} \alpha^{k-j} s_{j}+\gamma \delta \sum_{j=m}^{k-m} \alpha^{k-j} \tau^{j}+\delta \sum_{j=k-m+1}^{k-1} a_{k-j} \tau^{j} \\
& =\gamma \alpha^{k} s_{<m}\left(\frac{1}{\alpha}\right)+\gamma \delta \alpha^{m} \tau^{m} \sum_{j=0}^{k-2 m} \alpha^{k-2 m-j} \tau^{j}+\delta \sum_{j=1}^{m-1} a_{j} \tau^{k-j} \\
& =\gamma \alpha^{k} s_{<m}\left(\frac{1}{\alpha}\right)+\gamma \delta \alpha^{m} \tau^{m} \frac{\tau^{k-2 m+1}-\alpha^{k-2 m+1}}{\tau-\alpha}+\delta \tau^{k} a_{<m}\left(\frac{1}{\tau}\right) \\
& =\gamma \alpha^{k}\left(s_{<m}\left(\frac{1}{\alpha}\right)-\delta\left(\frac{\tau}{\alpha}\right)^{m} \frac{\alpha}{\tau-\alpha}\right)+\delta \tau^{k}\left(a_{<m}\left(\frac{1}{\tau}\right)+\gamma\left(\frac{\alpha}{\tau}\right)^{m} \frac{\tau}{\tau-\alpha}\right) \\
& \leqslant \gamma \alpha^{k} \cdot 0+\delta \tau^{k} \cdot 1=\delta \tau^{k}
\end{aligned}
$$

using (2) (with $\tau$ for $\sigma$ ) and the definition of $\delta$. Thus $s_{k}=O\left(\tau^{k}\right)$ and $s_{k}=o\left(\sigma^{k}\right)$. Under hypothesis (4), we get $\delta=s_{n} \tau^{-n}$ for some $n$ with $m \leqslant n<2 m$, and $s_{k} \leqslant \delta \tau^{k}=s_{n} \tau^{k-n}$ for $k \geqslant m$.

Again, it is not hard to see that the bounds provided by Lemma 13 cannot be improved essentially:

Corollary 14 Assume $0<\alpha<\sigma$ and $a_{k}=O\left(\alpha^{k}\right)$. Then the following statements are equivalent:

(a) $a\left(\frac{1}{\sigma}\right)<1$.

(b) $a_{<m}\left(\frac{1}{\sigma}\right)<1-\gamma\left(\frac{\alpha}{\sigma}\right)^{m} \frac{\sigma}{\sigma-\alpha}$ for some $m$ and $\gamma \geqslant \sup _{k \geqslant m} \frac{a_{k}}{\alpha^{k}}$.

(c) $s_{k}=O\left(\tau^{k}\right)$ for some $\tau<\sigma$.

(d) For some $\tau^{\prime}<\sigma$, eventually $\sqrt[k]{s_{k}}<\tau^{\prime}$.

(e) $\limsup \sqrt[k]{s_{k}}<\sigma$ 
Proof. For $(\mathrm{a}) \Longrightarrow(\mathrm{b})$, first find some $\gamma>0$ so that $a_{k} \leqslant \gamma \alpha^{k}$ for all $k$. As $\lim a_{<m}\left(\frac{1}{\sigma}\right)=$ $a\left(\frac{1}{\sigma}\right)<1$, there exists an $n$ such that for all $m \geqslant n$, we have $a_{<m}\left(\frac{1}{\sigma}\right)<\frac{1}{2}\left(1+a\left(\frac{1}{\sigma}\right)\right)$. Now choose an $m \geqslant n$ with $\gamma\left(\frac{\alpha}{\sigma}\right)^{m} \frac{\sigma}{\sigma-\alpha} \leqslant \frac{1}{2}\left(1-a\left(\frac{1}{\sigma}\right)\right)$. Then

$$
a_{<m}\left(\frac{1}{\sigma}\right)<\frac{1}{2}\left(1+a\left(\frac{1}{\sigma}\right)\right)=1-\frac{1}{2}\left(1-a\left(\frac{1}{\sigma}\right)\right) \leqslant 1-\gamma\left(\frac{\alpha}{\sigma}\right)^{m} \frac{\sigma}{\sigma-\alpha} .
$$

(b) $\Longrightarrow(\mathrm{c})$ follows from Lemma 13 .

$(\mathrm{c}) \Longrightarrow(\mathrm{d})$ : Choose $\varepsilon>0$ with $\tau^{k} \geqslant \varepsilon s_{k}$ for all $k$, then $\tau^{\prime}$ with $\sigma>\tau^{\prime}>\tau$, and finally $n$ with $\varepsilon>\left(\frac{\tau}{\tau^{\prime}}\right)^{n}$. Then each $k \geqslant n$ satisfies $\tau \geqslant \sqrt[k]{\varepsilon s_{k}}>\frac{\tau}{\tau^{\prime}} \sqrt[k]{s_{k}}$, hence $\tau^{\prime}>\sqrt[k]{s_{k}}$.

$(\mathrm{d}) \Longrightarrow(\mathrm{e}): \lim \sup \sqrt[k]{s_{k}} \leqslant \tau^{\prime}<\sigma$.

$(\mathrm{e}) \Longrightarrow(\mathrm{a})$ : Cauchy-Hadamard gives $\frac{1}{\sigma}<\varrho_{s} \leqslant \varrho_{a}$, hence $s\left(\frac{1}{\sigma}\right)\left(1-a\left(\frac{1}{\sigma}\right)\right)=1$ and therefore $a\left(\frac{1}{\sigma}\right)<1$.

Corollary 15 If $0<\varrho$ then $a(\varrho) \leqq 1 \Longleftrightarrow \varrho \leqq \varrho_{s}$.

The practical application of our lemmas is based on the following

Proposition 16 Let $m_{0}$ be a natural number so that $a_{k_{i}} \neq 0$ for some $k_{1}, \ldots, k_{u}<m_{0}$ with $\operatorname{gcd}\left(k_{1}, \ldots, k_{u}\right)=1$ and $a_{k} \leqslant \gamma \alpha^{k}$ for all $k \geqslant m_{0}$ (with $\alpha, \gamma>0$ fixed). Let

$$
\begin{aligned}
& \underline{a}_{m}(x)=x^{m-1}-\sum_{j=0}^{m-2} a_{m-j-1} x^{j} \text { and } \\
& \bar{a}_{m}(x)=\underline{a}_{m}(x)(x-\alpha)-\gamma \alpha^{m} .
\end{aligned}
$$

Then, for each $m \geqslant m_{0}$, there is a unique solution $\underline{\sigma}_{m}>0$ of the equation $\underline{a}_{m}(x)=0$ and a unique solution $\bar{\sigma}_{m}>\alpha$ of $\bar{a}_{m}(x)=0$. Furthermore,

$$
\begin{aligned}
& \underline{\sigma}_{m}^{k} \leqslant s_{k} \leqslant \bar{\sigma}_{m}^{k} \text { eventually, and } \\
& \underline{\sigma}_{m} \leqslant \underline{\sigma}_{m+1} \leqslant \lim \underline{\sigma}_{k}=\lim \sqrt[k]{s_{k}}=\frac{1}{\varrho_{s}} \leqslant \bar{\sigma}_{m+1} \leqslant \bar{\sigma}_{m} .
\end{aligned}
$$

Proof. For $m \geqslant m_{0}$, the polynomial $a_{<m}(x)$ is not zero. The equation $x^{1-m} \underline{a}_{m}(x)=$ $1-a_{<m}\left(\frac{1}{x}\right)=0$ has a unique positive solution $\underline{\sigma}_{m}$, as $1-a_{<m}\left(\frac{1}{x}\right)$ is strictly increasing in $x$, with $1-a_{<m}\left(\frac{1}{x}\right) \rightarrow 1$ for $x \rightarrow \infty$ and $1-a_{<m}\left(\frac{1}{x}\right) \rightarrow-\infty$ for $x \rightarrow 0^{+}$. Of course, $\underline{\sigma}_{m}$ is then also the unique positive root of $\underline{a}_{m}(x)$. Moreover,

$$
\underline{a}_{m+1}(x)=x^{m}\left(1-a_{<m}\left(\frac{1}{x}\right)\right)-a_{m}
$$

yields $\underline{a}_{m+1}\left(\underline{\sigma}_{m}\right)=-a_{m} \leqslant 0$, and as $\underline{a}_{m+1}(x) \rightarrow \infty$ for $x \rightarrow \infty$, it follows that $\underline{\sigma}_{m} \leqslant$ $\underline{\sigma}_{m+1}$. For $0<\sigma<\underline{\sigma}_{m}$, we have $a\left(\frac{1}{\sigma}\right)>1$, so that from Corollary 12 we know that $\sigma^{k} \leqslant s_{k}$ eventually. Hence, $\sigma \leqslant \lim \sup \sqrt[k]{s_{k}}=\frac{1}{\varrho_{s}}$ and, taking the limit $\sigma \rightarrow \underline{\sigma}_{m}$, also $\underline{\sigma}_{m} \leqslant \frac{1}{\varrho_{s}}$. For the equation $\lim \underline{\sigma}_{k}=\frac{1}{\varrho_{s}}$, it remains to show that $s(x)$ converges for 
$x<x_{0}:=\left(\lim \underline{\sigma}_{k}\right)^{-1}=\inf \frac{1}{\underline{\sigma}_{k}}$. Since $a_{<m}\left(x_{0}\right) \leqslant a_{<m}\left(\frac{1}{\underline{\sigma}_{m}}\right)=1$ for all $m$ and $a(x)$ is strictly increasing, we have $a(x)<a\left(x_{0}\right) \leqslant 1$, so that $s(x)=\sum^{m} a(x)^{n}$ converges to $(1-a(x))^{-1}$.

Similarly, for $x>\alpha$, the function

$$
h_{m}(x)=\frac{\bar{a}_{m}(x)}{x^{m}-\alpha x^{m-1}}=1-a_{<m}\left(\frac{1}{x}\right)-\gamma\left(\frac{\alpha}{x}\right)^{m} \frac{x}{x-\alpha}
$$

is strictly increasing because $a_{<m}\left(\frac{1}{x}\right), \gamma\left(\frac{\alpha}{x}\right)^{m}$ and $\frac{x}{x-\alpha}$ are strictly decreasing functions. As $h_{m}(x) \rightarrow-\infty$ for $x \rightarrow \alpha^{+}$and $h_{m}(x) \rightarrow 1$ for $x \rightarrow \infty$, there is a unique solution $\bar{\sigma}_{m}>\alpha$ of $h_{m}(x)=0$, and this is also the unique solution of $\bar{a}_{m}(x)=0(x>\alpha)$. Furthermore, substitution of $\bar{\sigma}_{m}$ for $x$ in the equation

$$
\begin{aligned}
\bar{a}_{m+1}(x) & =x^{m}\left(1-a_{<m}\left(\frac{1}{x}\right)-a_{m} x^{-m}\right)(x-\alpha)-\gamma \alpha^{m+1} \\
& =x \bar{a}_{m}(x)+\left(\gamma \alpha^{m}-a_{m}\right)(x-\alpha)
\end{aligned}
$$

gives $\bar{a}_{m+1}\left(\bar{\sigma}_{m}\right)=\left(\gamma \alpha^{m}-a_{m}\right)\left(\bar{\sigma}_{m}-\alpha\right) \geqslant 0$ because of $a_{m} \leqslant \gamma \alpha^{m}$. As before, we conclude that $\bar{\sigma}_{m+1} \leqslant \bar{\sigma}_{m}$ since $\bar{a}_{m+1}(\alpha)=-\gamma \alpha^{m+1}<0$. Now $\bar{a}_{m}(x) \rightarrow \infty$ for $x \rightarrow \infty$ implies for $\sigma>\bar{\sigma}_{m}$ :

$$
\begin{aligned}
0<\bar{a}_{m}(\sigma) & =\underline{a}_{m}(\sigma)(\sigma-\alpha)-\gamma \alpha^{m} \\
& =\sigma^{m-1}\left(1-a_{<m}\left(\frac{1}{\sigma}\right)\right)(\sigma-\alpha)-\gamma \alpha^{m},
\end{aligned}
$$

and as $\alpha$ lies between 0 and $\sigma$, the previous inequality is equivalent to (2) in Lemma 13, whence $s_{k} \leqslant \sigma^{k}$ eventually. Thus $\frac{1}{\varrho_{s}}=\lim \sup \sqrt[k]{s_{k}} \leqslant \sigma$ and finally also $\frac{1}{\varrho_{s}} \leqslant \bar{\sigma}_{m}$.

In all, we see that full information about the coefficients $a_{j}(j<m)$ provides a twosided asymptotical estimate

$$
\underline{\sigma}_{m}<\lim \sqrt[k]{s_{k}}<\bar{\sigma}_{m}
$$

If the numbers $a_{j}$ are known even for $j<2 m$ then so are the numbers $s_{j}$, and one obtains from the proofs of Lemmas 9 and 13 concrete estimates

$$
\begin{aligned}
\underline{\delta}_{m} \underline{\sigma}_{m}^{k} \leqslant s_{k} \leqslant \bar{\delta}_{m} \bar{\sigma}_{m}^{k} \text { for } k \geqslant m, \text { with } \\
\underline{\delta}_{m}=\min \left\{s_{j} \underline{\sigma}_{m}^{-j}: m \leqslant j<2 m\right\}, \\
\bar{\delta}_{m}=\max \left\{s_{j} \bar{\sigma}_{m}^{-j}: m \leqslant j<2 m\right\},
\end{aligned}
$$

the upper bound requiring that $\alpha^{m-1}\left(\bar{\sigma}_{m}-\alpha\right) s_{<m}\left(\frac{1}{\alpha}\right) \leqslant s_{m}$. Note that, for the upper bound, the hardest part may often be to determine $\alpha$ and $\gamma$ so that $a_{k} \leqslant \gamma \alpha^{k}$ at least for all $k \geqslant m$.

\section{$5 \quad$ Distributive lattices with less than 50 elements}

For efficient applications of the theory developed in the previous sections, one needs sufficiently many of the numbers $v_{k}$ and $w_{k}$ for small $k$. We determined $v_{k}$ for $k \leqslant 49$ with the orderly algorithm described in $[8,9]$. The numbers $d_{k}$ and $w_{k}$ are then obtained by Corollaries 2 and 7, the results are shown in Table 1 and Figure 3. Seeking a good fit, one may approximate these values in the following form: 
Proposition 17 For $k<50$,

$$
\begin{aligned}
d_{k} & =1.8439^{k-4}\left(1+\delta_{k}\right), & & 0 \leqslant \delta_{k}<(7 / k)^{3}, \\
v_{k} & =1.7250^{k-8}\left(1+(-1)^{k} \gamma_{k}\right), & & 0 \leqslant \gamma_{k}<(14 / k)^{3}, \\
w_{k} & =1.6765^{k-8}\left(1-\beta_{k}\right), & & 0 \leqslant \beta_{k}<(23 / k)^{3},
\end{aligned}
$$

except for $k \in\{2,8\}$ in the third case.

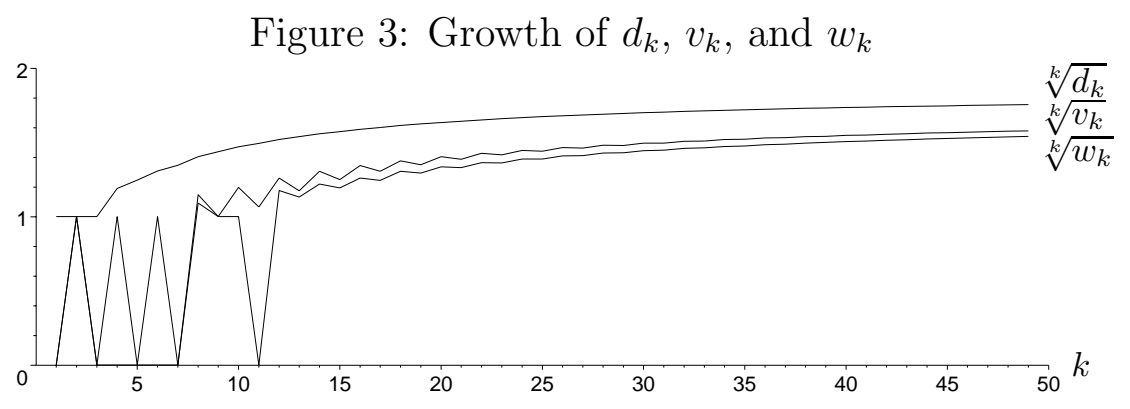

\section{Lower and upper bounds for $v_{k}$ and $d_{k}$}

We are now going to apply the general results established in Section 4 to the two cases that concern us here, viz.

(1) $a_{k}=v_{k+1}$, the number of all ordinally indecomposable posets with $k$ nonempty antichains, or, equivalently, the number of all vertically indecomposable distributive lattices with $k+1$ elements, and

$s_{k}=d_{k+1}$, the number of all posets with $k$ nonempty antichains, respectively, of all distributive lattices with $k+1$ elements.

(2) $a_{k}=w_{k}$, the number of all canonically 2-indecomposable posets with $k$ antichains, and

$s_{k}=v_{k}$, the number of all ordinally indecomposable posets with $k$ antichains.

Before we turn to numerical evaluation, let us note a few qualitative results that do not require any concrete calculation of the involved numbers.

We know that, in both cases, $\left(s_{k}\right)$ is the summatorial sequence of $\left(a_{k}\right)$. Thus, $s(x)=$ $(1-a(x))^{-1}$, and as $a(x)=x v(x)$ and $d(x)=1+x s(x)$ in the first case, we get

$$
d(x)=1+\frac{x}{1-x v(x)},
$$

and in the second case,

$$
v(x)=\frac{1}{1-w(x)}
$$


Table 1: Numbers of [vertically indecomposable / canonically 2-indecomposable] distributive lattices

\begin{tabular}{rrrr}
\hline$k$ & $d_{k}$ & $v_{k}$ & $w_{k}$ \\
\hline 1 & 1 & 0 & 0 \\
2 & 1 & 1 & 1 \\
3 & 1 & 0 & 0 \\
4 & 2 & 1 & 0 \\
5 & 3 & 0 & 0 \\
6 & 5 & 1 & 0 \\
7 & 8 & 0 & 0 \\
8 & 15 & 3 & 2 \\
9 & 26 & 1 & 1 \\
10 & 47 & 6 & 1 \\
11 & 82 & 2 & 0 \\
12 & 151 & 16 & 7 \\
13 & 269 & 8 & 5 \\
14 & 494 & 42 & 16 \\
15 & 891 & 28 & 14 \\
16 & 1639 & 112 & 40 \\
17 & 2978 & 93 & 41 \\
18 & 5483 & 311 & 120 \\
19 & 10006 & 295 & 131 \\
20 & 18428 & 869 & 321 \\
21 & 33749 & 939 & 402 \\
22 & 62162 & 2454 & 901 \\
23 & 114083 & 2931 & 1210 \\
24 & 210189 & 7032 & 2590 \\
25 & 386292 & 9036 & 3621 \\
26 & 711811 & 20301 & 7371 \\
27 & 1309475 & 27701 & 10841 \\
28 & 2413144 & 58929 & 21178 \\
29 & 4442221 & 84413 & 32222 \\
30 & 8186962 & 172104 & 61273 \\
31 & 15077454 & 255919 & 95408 \\
32 & 27789108 & 504637 & 177384 \\
33 & 51193086 & 773511 & 282405 \\
34 & 94357143 & 1484392 & 515174 \\
35 & 173859936 & 2331180 & 833295 \\
36 & 320462062 & 4378773 & 1500030 \\
37 & 590555664 & 7009288 & 2455337 \\
38 & 1088548290 & 12944347 & 4372535 \\
39 & 2006193418 & 21039961 & 7229231 \\
40 & 3697997558 & 38328890 & 12761691 \\
41 & 6815841849 & 63067623 & 21260746 \\
42 & 12563729268 & 113651785 & 37286778 \\
43 & 23157428823 & 188831922 & 62483221 \\
44 & 42686759863 & 337361112 & 109014426 \\
45 & 78682454720 & 564890985 & 183542099 \\
46 & 145038561665 & 1002268019 & 318906720 \\
47 & 267348052028 & 1688673026 & 538889399 \\
48 & 492815778109 & 2979703035 & 933361886 \\
49 & 908414736485 & 5045200597 & 1581666042 \\
\hline & & &
\end{tabular}


Theorem 18 For $k \geqslant 8$, there are at least $1.678^{k-10}$ many unlabeled vertically indecomposable distributive lattices of size $k$ and dimension $\leqslant 3$.

Proof. We inductively define systems $\mathcal{V}_{k}$ of $k$-element subsets of the distributive lattice $(\omega, \leqslant)^{3}$. Let $[a, b]=\{a, a+1, \ldots, b\}$, and write $\left(x_{A}, y_{A}, z_{A}\right)$ for the pointwise maximum of a subset $A \subseteq \omega^{3}$ (if it exists). Put

$$
\mathcal{V}_{6}=\{[0,1] \times[0,2] \times[0,0],[0,2] \times[0,1] \times[0,0]\}
$$

and $\mathcal{V}_{k}=\emptyset$ for $k \in\{1,2,3,4,5,7\}$. For $k \geqslant 8$, let $\mathcal{V}_{k}$ be the smallest system such that

$$
A^{(\xi, \eta, \zeta)}=A \cup\left(\left[x_{A}-1, x_{A}+\xi\right] \times\left[y_{A}-1, y_{A}+\eta\right] \times\left[z_{A}, z_{A}+\zeta\right]\right) \in \mathcal{V}_{k}
$$

whenever (i) $\xi, \eta, \zeta \in \omega$, (ii) $(\xi+\eta)(\eta+\zeta)(\zeta+\xi)>0$ or $\xi+\eta+\zeta=1$, and (iii) $A \in \mathcal{V}_{k-\delta}$, where $\delta=(\xi+2)(\eta+2)(\zeta+1)-4$. In other words, we construct larger subsets from smaller ones by replacing the top square $\left[x_{A}-1, x_{A}\right] \times\left[y_{A}-1, y_{A}\right] \times\left\{z_{A}\right\}$ with some larger cuboid. As is easily seen, condition (ii) assures that, for each $A^{\prime} \in \mathcal{V}_{k}$, there is exactly one quadruple $(A, \xi, \eta, \zeta)$ with $A^{\prime}=A^{(\xi, \eta, \zeta)}$. By construction, each $A \in \mathcal{V}_{k}$ is a sublattice of $(\omega, \leqslant)^{3}$, hence distributive (cf. Section 2). The unique other lattice $A^{\prime} \in \mathcal{V}_{k}$ that is isomorphic to $A$ is the lattice $A^{\prime}=\{(y, x, z):(x, y, z) \in A\}$. For $\delta=2,4,5,8,11$, or 12, there are exactly $2,1,1,4,2$, or 6 possibilities for $(\xi, \eta, \zeta)$, respectively, so that

$$
\left|\mathcal{V}_{k}\right| \geqslant 2\left|\mathcal{V}_{k-2}\right|+\left|\mathcal{V}_{k-4}\right|+\left|\mathcal{V}_{k-5}\right|+4\left|\mathcal{V}_{k-8}\right|+2\left|\mathcal{V}_{k-11}\right|+6\left|\mathcal{V}_{k-12}\right|
$$

for $k \geqslant 13$, and $\left|\mathcal{V}_{k}\right| \geqslant 2 \cdot 1.678^{k-10}$ for $14 \leqslant k \leqslant 25$. Hence also $\left|\mathcal{V}_{k}\right| \geqslant 2 \cdot 1.678^{k-10}$ for $k \geqslant 26$. For $8 \leqslant k \leqslant 13$, the proposition is verified directly.

The representation of an isomorphism type by an increasing sequence $0=z_{1} \leqslant z_{2} \leqslant$ $\cdots \leqslant z_{n} \leqslant k-2$ (for $k \geqslant 2$ ) instantly provides us with an exponential upper bound on $d_{k}$. Making the sequences strictly increasing,

$$
1 \leqslant z_{2}+1<z_{3}+2<\cdots<z_{n}+(n-1) \leqslant(k-2)+(n-1),
$$

we get

$$
d_{k} \leqslant \sum_{n=1}^{k}\left(\begin{array}{c}
k+n-3 \\
n-1
\end{array}\right)=\left(\begin{array}{c}
2 k-2 \\
k-1
\end{array}\right)<4^{k-1} .
$$

One can improve this upper bound by considering vertically indecomposable lattices first. Such lattices don't have "knots", i.e. nonextremal elements comparable to all other elements; thus, each step of the doubling construction must give at least two new elements. Hence, $2 n \leqslant k, z_{2}=0$, and $z_{n} \leqslant k-4$. Therefore, putting $\ell=\lfloor k / 2\rfloor, v_{k}$ satisfies

$$
v_{k} \leqslant \sum_{n=2}^{\ell}\left(\begin{array}{c}
k+n-6 \\
n-2
\end{array}\right)=\left(\begin{array}{c}
k+\ell-5 \\
\ell-2
\end{array}\right) \quad(k \geqslant 3) .
$$


This easily gives the following exponential bound:

$$
v_{k} \leqslant \frac{\alpha^{k}}{25 \sqrt{k}}=o\left(\alpha^{k}\right) \quad \text { for } \alpha=\frac{3}{2} \sqrt{3}<2.6, k \neq 2 .
$$

But we can do better:

Theorem 19 The numbers $v_{\leqslant k}$ of vertically indecomposable distributive lattices with at most $k$ elements satisfy the inequalities

$$
v_{k} \leqslant v_{\leqslant k} \leqslant \sum_{t=1}^{\lfloor k / 2\rfloor-1}\left(\begin{array}{c}
k-4 \\
t-1
\end{array}\right)\left(\begin{array}{c}
\lfloor k / 4+t / 2\rfloor \\
t
\end{array}\right)<2.33^{k-4},
$$

and $v_{\leqslant k}=o\left(2.33^{k}\right)$.

Proof. We know that the vertically indecomposable distributive lattices of height $n$ and size $\leqslant k$ may be coded by certain integer sequences $\left(z_{1}, \ldots, z_{n}\right)$ with

$$
0=z_{1}=z_{2} \leqslant \cdots \leqslant z_{n} \leqslant k-4 .
$$

Moreover, if $z_{i}=z_{i+1}$ then the interval doubled at step $i>1$ is doubled again at step $i+1$, so that at least four elements must be added in the latter case. (More generally, if $z_{i}=z_{i+1}=\cdots=z_{i+r}$ then at least $2^{j+1}$ elements have to be added at step $i+j, j \leqslant r$. Denoting by $s$ the number of indices $i$ with $z_{i}=z_{i+1}$, we finally have generated at least $2+2(n-1)+2 s$ elements, i.e.

$$
2 n+2 s \leqslant k \quad(\star)
$$

There are $\left(\begin{array}{c}n-2 \\ s\end{array}\right)$ possibilities to choose $s$ places $i$ with $1<i<n$ and $z_{i}=z_{i}+1$. For the remaining $t=n-1-s$ indices $i_{1}<\cdots<i_{t}$, there are $\left(\begin{array}{c}k-4 \\ t-1\end{array}\right)$ many strictly increasing sequences $0=z_{i_{1}}<\cdots<z_{i_{t}} \leqslant k-4$. By $(\star)$, we have the inequalities $t<n \leqslant \frac{k}{2}-s=\frac{k}{2}-n+1+t$, hence

$$
0<t<n \leqslant \frac{k}{4}+\frac{t}{2}+\frac{1}{2} .
$$

In all, this gives

$$
\begin{aligned}
v_{k} \leqslant v_{\leqslant k} & \leqslant \sum_{t=1}^{\lfloor k / 2\rfloor-1}\left(\begin{array}{c}
k-4 \\
t-1
\end{array}\right) \sum_{n=t+1}^{\lfloor k / 4+t / 2+1\rfloor}\left(\begin{array}{c}
n-2 \\
n-t-1
\end{array}\right) \\
& =\sum_{t=1}^{\lfloor k / 2\rfloor-1}\left(\begin{array}{c}
k-4 \\
t-1
\end{array}\right)\left(\begin{array}{c}
\lfloor k / 4+t / 2\rfloor \\
t
\end{array}\right) \\
& \leqslant \sum_{t=1}^{\lfloor k / 2\rfloor-1} \frac{1}{2}\left(\begin{array}{c}
k \\
t
\end{array}\right)\left(\begin{array}{c}
\lfloor k / 4+t / 2\rfloor \\
t
\end{array}\right) \\
& <\frac{k}{4} \max \left\{\left(\begin{array}{l}
k \\
t
\end{array}\right)\left(\begin{array}{c}
\lfloor k / 4+t / 2\rfloor \\
t
\end{array}\right): 1 \leqslant t \leqslant \frac{k}{2}-1\right\} .
\end{aligned}
$$


Now, the inequalities

$$
\left(\begin{array}{c}
\lfloor\alpha t\rfloor \\
t
\end{array}\right) \leqslant \min \left\{1, \sqrt{\frac{\alpha}{2 \pi(\alpha-1) t}}\right\}\left(\alpha^{\alpha}(\alpha-1)^{1-\alpha}\right)^{t},
$$

which follow from the known estimate $n !=(2 \pi n)^{-1 / 2}\left(\frac{n}{e}\right)^{n} e^{\frac{1}{12 n+\varepsilon}}$ with $0 \leqslant \varepsilon \leqslant \frac{1}{4}$ (cf. [10], p. 355), yield for $\alpha=\frac{k}{t}>2$ :

$$
\left(\begin{array}{l}
k \\
t
\end{array}\right)\left(\begin{array}{c}
\lfloor k / 4+t / 2\rfloor \\
t
\end{array}\right) \leqslant \min \left\{1, \frac{g(\alpha)}{k}\right\} f(\alpha)^{k}
$$

with

$$
\begin{aligned}
f(\alpha) & =\left(\alpha^{\alpha}(\alpha-1)^{1-\alpha}\left(\frac{\alpha}{4}+\frac{1}{2}\right)^{\frac{\alpha}{4}+\frac{1}{2}}\left(\frac{\alpha}{4}-\frac{1}{2}\right)^{\frac{1}{2}-\frac{\alpha}{4}}\right)^{\frac{1}{\alpha}} \\
& =\alpha(\alpha-1)^{\frac{1}{\alpha}-1}\left(\frac{\alpha}{4}+\frac{1}{2}\right)^{\frac{1}{2 \alpha}+\frac{1}{4}}\left(\frac{\alpha}{4}-\frac{1}{2}\right)^{\frac{1}{2 \alpha}-\frac{1}{4}} \text { and } \\
g(\alpha) & =\alpha \sqrt{\frac{\alpha}{2 \pi(\alpha-1)}} \sqrt{\frac{\alpha+2}{2 \pi(\alpha-2)}}=\frac{\alpha}{2 \pi} \sqrt{\frac{\alpha(\alpha+2)}{(\alpha-1)(\alpha-2)}} .
\end{aligned}
$$

Numerical evaluation yields

$$
f(\alpha)<2.3295<2.33 \text { for all } \alpha>2 \text {, }
$$

providing already the asymptotical result

$$
v_{\leqslant k}=o\left(2.33^{k}\right)
$$

To obtain the explicit estimate $v_{\leqslant k} \leqslant 2.33^{k-4}$ for all $k$, one has to be more careful. Putting

$$
\begin{aligned}
f_{l}:=f\left(0.27^{-1}\right)=\max \left\{f(\alpha): \alpha \geqslant 0.27^{-1}\right\}, \\
f_{r}:=f\left(0.41^{-1}\right)=\max \left\{f(\alpha): 2 \leqslant \alpha \leqslant 0.41^{-1}\right\}, \\
\bar{f}_{n}:=\max \left\{f(\alpha): \frac{100}{n+1} \leqslant \alpha \leqslant \frac{100}{n}\right\} \quad(n<50), \\
\bar{g}_{n}:=\max \left\{g(\alpha): \frac{100}{n+1} \leqslant \alpha \leqslant \frac{100}{n}\right\} \quad(n<50),
\end{aligned}
$$

we get

$$
\begin{aligned}
v_{\leqslant k} & \leqslant \frac{1}{2}\left(\sum_{t<0.27 k} f_{l}{ }^{k}+\sum_{n=27}^{41} \sum_{\frac{n k}{100} \leqslant t \leqslant \frac{(n+1) k}{100}} \bar{f}_{n}^{k} \bar{g}_{n} k^{-1}+\sum_{0.42 k \leqslant t<0.5 k} f_{r}{ }^{k}\right) \\
& \leqslant 0.135 k \cdot 2.267^{k}+\frac{1}{200} \sum_{n=27}^{41} \bar{f}_{n}^{k} \bar{g}_{n}+0.04 k \cdot 2.262^{k} .
\end{aligned}
$$


Hence, for $k \geqslant 400$,

$$
\begin{aligned}
2.33^{-k} v_{\leqslant k} & \leqslant 54\left(\frac{2.267}{2.33}\right)^{400}+\frac{1}{200} \sum_{n=27}^{41}\left(\frac{\bar{f}_{n}}{2.33}\right)^{400} \cdot \bar{g}_{n}+16\left(\frac{2.262}{2.33}\right)^{400} \\
& \leqslant 0.001+0.031+0.001=0.033<2.33^{-4} .
\end{aligned}
$$

For $k<400$, the upper bound $2.33^{k-4}$ may be checked directly.

With more effort, this upper bound can be improved considerably (at least to $2.28^{k}$ ) by taking into account the remark about the increment $2^{j+1}$ at step $i+j$, which restricts the possibilities for the coding sequences enormously. However, even $2.28^{k}$ seems to be a very rough upper bound, since $v_{k}<1.8^{k}$ for all $k<50$ (see also Proposition 17). Nevertheless, we shall need the bound $2.33^{k-4}$ (not only $2.33^{k}$ ) below for an estimate of the $d_{k}$. We apply Proposition 16 to the following data:

(1) $a_{k}=v_{k+1}, m_{0}=2\left(v_{2}=1\right), \alpha=2.33$, and $\gamma=2.33^{-4}$.

(2) $a_{k}=w_{k}, m_{0}=10\left(w_{2}=w_{9}=1, \operatorname{gcd}(2,9)=1\right)$.

In case (1), we get:

Theorem 20 Let $m$ be a fixed natural number, and denote by $\underline{\sigma}_{m}$ and $\bar{\sigma}_{m}$ the unique positive roots of the equations

$$
x^{m-1}-\sum_{j=2}^{m} v_{j} x^{m-j}=0 \quad \text { resp. } \quad\left(x^{m-1}-\sum_{j=2}^{m} v_{j} x^{m-j}\right)(x-\alpha)=\gamma \alpha^{m} .
$$

Then ${\underline{\sigma_{m}}}_{m}^{k}=O\left(d_{k}\right), d_{k}=O\left(\bar{\sigma}_{m}^{k}\right)$, and $\underline{\sigma}_{m} \leqslant \sqrt[k]{d_{k}} \leqslant \bar{\sigma}_{m}$ eventually. Moreover, $\lim \sqrt[k]{s_{k}}=$ $\lim \underline{\sigma}_{k}$.

More explicitly, put

$$
\begin{aligned}
\underline{\delta}_{m} & =\min \left\{d_{j} \underline{\sigma}_{m}^{-j}: m<j \leqslant 2 m\right\} \quad \text { and } \\
\bar{\delta}_{m} & =\max \left\{d_{j} \bar{\sigma}_{m}^{-j}: m<j \leqslant 2 m\right\} .
\end{aligned}
$$

Then $d_{k} \geqslant \underline{\delta}_{m} \underline{\sigma}_{m}^{k}$ for $k \geqslant m$, and under the proviso that

$$
d_{m+1} \geqslant \alpha^{m-1}\left(\bar{\sigma}_{m}-\alpha\right) \sum_{j=1}^{m} d_{j} \alpha^{-j+1}
$$

(which holds for $m \leqslant 4$ ), $d_{k} \leqslant \bar{\delta}_{m} \bar{\sigma}_{m}^{k}$. Numerical evaluation yields

$$
\begin{aligned}
& \underline{\sigma}_{24}>1.81, \underline{\delta}_{24}>\frac{1}{4}, \quad \text { hence } d_{k}>\frac{1}{4} 1.81^{k} \text { for } k \geqslant 24, \\
& \underline{\sigma}_{49}>1.8388, \\
& \underline{\sigma}_{60}>1.84\left(\text { using } v_{k} \geqslant \sum_{j=k-49}^{k-1} w_{k-j} v_{j} \text { for } k \geqslant 50\right), \\
& \bar{\sigma}_{4}<2.46, \bar{\delta}_{4}<0.34, \quad \text { hence } d_{k}<0.34 \cdot 2.46^{k}, \\
& \bar{\sigma}_{49}<2.385<2.39, \quad \text { hence } d_{k}=o\left(2.39^{k}\right) .
\end{aligned}
$$


Figure 4: Exponential bounds on $d_{k}$

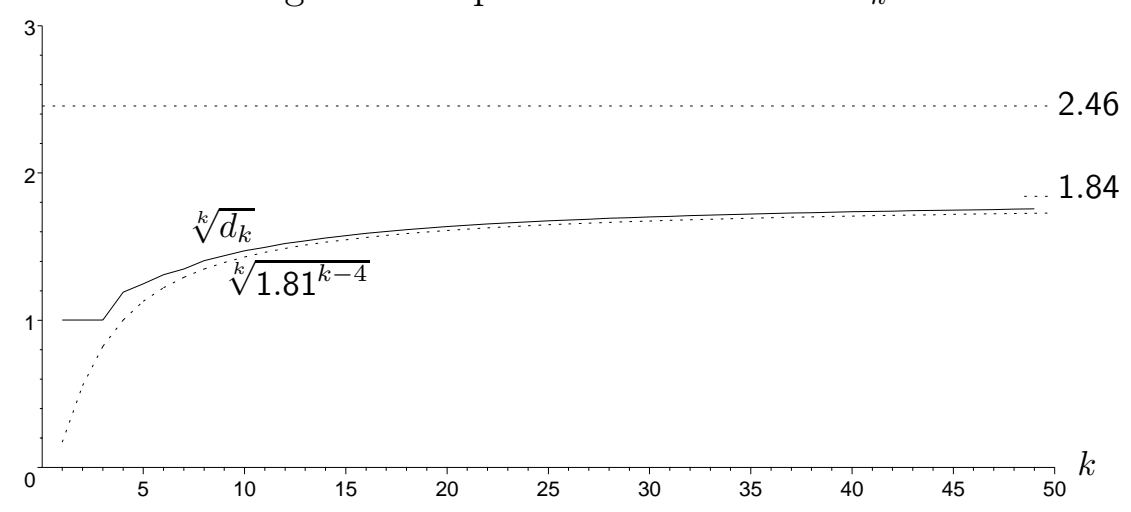

Corollary 21 (Fig. 4)

$1.81^{k-4}<d_{k}<2.46^{k-1}$ for all $k$, and $1.84^{k}<d_{k}<2.39^{k}$ eventually.

Similarly, in case (2) we obtain

Theorem 22 Let $\underline{\tau}_{m}$ denote the unique positive root of the equation

$$
x^{m-1}-\sum_{j=0}^{m-2} w_{m-j-1} x^{j} .
$$

and put $\nu_{m}=\min \left\{v_{j} \underline{\tau}_{m}^{-j}: m \leqslant j<2 m\right\}$. Then $v_{k} \geqslant \nu_{m} \underline{\tau}_{m}^{k}$ for $k \geqslant m$. Hence, for fixed $m, \underline{\tau}_{m}^{k}=O\left(v_{k}\right)$ and $\sqrt[k]{v_{k}} \geqslant \underline{\tau}_{m}$ eventually.

Numerical evaluation yields

$$
\begin{array}{ll}
\underline{\tau}_{24}>1.54, & \text { hence } v_{k} \geqslant v_{24} \cdot 1.54^{k-24} \text { for } k \geqslant 26, \\
\underline{\tau}_{50}>1.66>\underline{\tau}_{49}, & \text { hence } 1.66^{k}=o\left(v_{k}\right)
\end{array}
$$

Corollary 23 (Fig. 5)

$v_{k} \geqslant\left\lfloor 1.5^{k-9}\right\rfloor$ for all $k, v_{k} \geqslant 1.54^{k-4}$ for $k \geqslant 24$, and $v_{k} \geqslant 1.66^{k}$ eventually.

As we see, the base of the lower bound obtained here does not exceed the one from Theorem 18. However, the $w_{k}$ will probably serve for better bounds when more numerical material will be known.

Corollary $24\left(\frac{5}{3}\right)^{k-10} \leqslant v_{k} \leqslant\left(\frac{7}{3}\right)^{k-4}$ for all $k \geqslant 8$.

We conclude with some open questions:

(1) Is it true that $v_{k}=o\left(d_{k}\right)$ and $w_{k}=o\left(v_{k}\right)$ ?

(2) Can one even show that $\lim \sqrt[k]{w_{k}}<\lim \sqrt[k]{v_{k}}<\lim \sqrt[k]{d_{k}}$ ?

(3) How far can the upper bound be improved, e.g., is $d_{k} \leqslant 2^{k}$ ? 
Figure 5: Exponential bounds on $v_{k}$

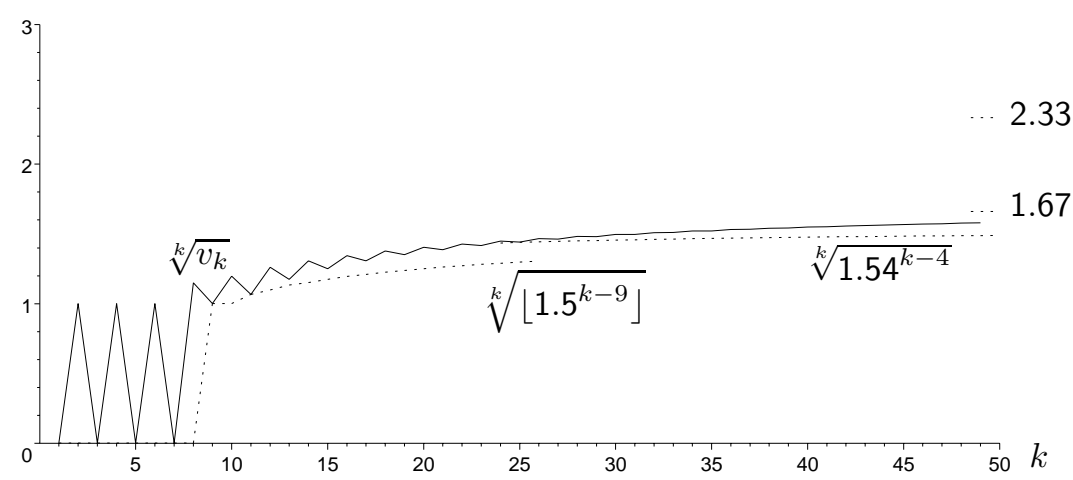

\section{References}

[1] P. Alexandroff, Diskrete Räume. Math. Sb. (N.S.) 2 (1937), 501-518.

[2] G. Birkhoff, Rings of sets. Duke Math. J. 3 (1937), 443-454.

[3] G. Birkhoff, Lattice Theory. Amer. Math. Soc. Coll. Publ. 25, $3^{\text {rd }}$ ed., Providence, R.I., 1973.

[4] A. Day, A simple solution to the word problem for lattices. Canad. Math. Bull. 13 (1970), 253-254.

[5] M. Erné, On the cardinalities of finite topologies and the number of antichains in partially ordered sets. Discrete Math. 35 (1981), 119-133.

[6] M. Erné, The number of partially ordered sets with more points than unrelated pairs. Discrete Math. 105 (1992), 49-60.

[7] M. Erné and K. Stege, Combinatorial applications of ordinal sum decompositions. Ars combinatoria 40 (1995), 65-88.

[8] J. Heitzig and J. Reinhold, Counting finite lattices, Tech. Report no. 298 (1999), Universität Hannover.

[9] J. Heitzig and J. Reinhold, The number of unlabeled orders on fourteen elements, 1999, to appear in Order.

[10] K. Königsberger, Analysis 1. Springer, Berlin-Heidelberg-New York, 1999. 